\title{
Enhancing the Teaching of Evolution in Public Health
}

\author{
Gilbert S. Omenn
}

Published online: 22 December 2011

(C) Springer Science+Business Media, LLC 2011

\begin{abstract}
Public health courses are emerging as popular undergraduate offerings, especially at universities with schools of public health. It is important to note that evolution has shaped the burden of disease in the modern world in which we practice and educate for public health. Human cultures and technologies have modified life on Planet Earth and have co-evolved with myriad other species, including microorganisms, plant and animal sources of food, invertebrate vectors of disease, and intermediate bird, mammal, and primate hosts. Molecular mechanisms of evolution have produced differential resistance or susceptibility to infectious agents, including malaria, plague, smallpox, TB, measles, and diarrheal and respiratory diseases. The domestication of sheep and cattle led to natural selection in favor of human populations able to digest milk throughout life through persistence into adulthood of lactase enzyme expression in the intestine, a major story of anthropology. The emergence of a "Western diet" of dairy, refined cereal grains, refined sugars, vegetable oils, alcoholic beverages, salt, and omega-6-rich meats has dramatically altered glycemic load, fatty acid composition, macronutrients, acid-base balance, sodium/potassium ratio, and fiber content. This is a major story of nutrition and disease. The results include epidemics of atherosclerotic cardiovascular disease, obesity, diabetes, high blood pressure, osteoporosis, certain cancers, and bowel, inflammatory, and autoimmune disorders. Another interesting phenomenon is the selection of excessive hemostatic activity from platelets and the plasma
\end{abstract}

G. S. Omenn $(\bowtie)$

University of Michigan,

Ann Arbor, MI 48109, USA

e-mail: gomenn@umich.edu clotting proteins; what was protective against death from bleeding after injuries among hunter-gatherers or from pregnancy-related hemorrhage now contributes to thrombosis underlying heart attacks and strokes. Conversely, there is little pressure against hemostasis and thrombosis since deaths from these causes occur mostly after the reproductive years of life. Learning about evolution over millennia for humans and over hours or days for microbes enlivens the experience of understanding evolutionary biology in public health context.

Keywords Evolutionary medicine · Public health · Evolution · Public health education · Lactase persistence . Western diets $\cdot$ Hemostasis $\cdot$ Microbes

\section{Introduction}

Public health education is evolving rapidly in the United States and around the world. It is ironic that medicine and healthcare services dominate thinking and expenditures for health in the United States, since a broader concept of the social, economic, biological, and technological determinants of health and disease would treat health care as a variable within public health. When I became dean of the School of Public Health \& Community Medicine at the University of Washington in 1982, there were 22 accredited schools of public health offering the Master of Public Health and related master's and doctoral degree programs and conducting research within the public health sciences. Now there are 48 schools, plus 76 institutions with accredited master's of public health programs in the United States. With greatly enhanced public and student interest in global health, many 
universities are introducing or expanding undergraduate courses in public health, including environmental health, nutrition and health, epidemiological surveillance of emerging infections, health policy, and behavioral and socioeconomic determinants of health. Internet communication is helping to democratize access to actionable knowledge in public health, including the SuperCourse of Global Health, launched by Ronald Laporte and colleagues at the University of Pittsburgh (www.pitt.edu/ super1), a network of an estimated 65,000 scientists in 174 countries sharing at no charge 5000 lectures in 31 languages; we have now created an expanded SuperCourse of Science, housed at the Bibliotheca Alexandrina in Egypt (Laporte et al. 2006).

Evolution and its elements of natural selection, population migration, genetic drift, and founder effects have shaped the human species and continue to do so (Vogel and Motulsky 1997; Stearns and Koella 2007; Trevathan et al. 2008; Gluckman et al. 2009; Omenn 2010). Meanwhile, evolving human cultures and their technologies have modified and co-evolved with other species. Some changes occur over numerous generations, even thousands of years, while others, especially involving microbes, including those in our own microbiome, are observable in hours or days.

Determinants of adult phenotypes reflect the concept of "evo-devo," the interaction of evolutionary and developmental processes (Stearns and Koella 2007). These processes are active early in development and influence phenotypes throughout the lifespan, maintaining or enhancing fitness on a timescale intermediate between selection and homeostasis. These manifestations of plasticity are acquired; some are reversible, others irreversible. These observations are matched at the molecular level by epigenetic modifications such as methylation of cytosines in the DNA and methylation, acetylation, and other modifications of histone proteins wrapped around the DNA in chromosomes. Plasticity and epigenetics link genes, development, and environment in the triangle of the determinants of phenotypes (Fig. 1).

\section{Genes}

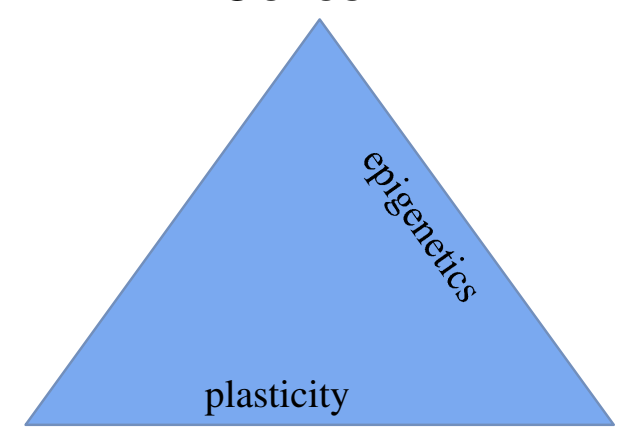

\section{Development}

Environment

Fig. 1 Roles of plasticity and epigenetics in linking genes, development, and environment to produce phenotypes
Exposures to chemical, physical, pharmaceutical, dietary, and microbial agents from environmental sources can affect in utero and postnatal development.

Drivers of evolution in the context of population health notably include:

- Infectious agents, including roles of intermediate hosts in nature, agriculture, and pets

- Dramatic changes in diet and physical activity

- Climate change and other diverse causes of migration of populations

Advances in population genetics and evolutionary biology have facilitated in-depth analyses of gene-environment interactions in human populations and other species. Public health research and public health practice focus on understanding, protecting, and enhancing the health of communities and populations. The substrates for the study of evolution in public health include international patterns of incidence and prevalence of disease, influences of human and animal behavior, and cultural changes in agriculture and food processing (Omenn 2010).

\section{Susceptibility and Resistance to Infectious Diseases}

There is ample evidence of the evolution of differential susceptibility to major infectious diseases (Vogel and Motulsky 1997; Ewald 2004). The best-known examples are two different types of malaria, due to Plasmodium falciparum and to Plasmodium vivax.

Malaria The protozoan parasite $P$. falciparum causes the most severe form of malaria, with more than one million deaths annually worldwide. Rates of malaria infection are correlated with the distribution of the obligate intermediate host, Anopheles mosquitoes that multiply in stagnant water, a situation driven by agricultural practices involving deforestation both historically and currently. The most obvious ways to avoid malaria infection are migration away from geographic areas with high prevalence of Anopheles and Plasmodium and elimination of the host with antimalarial chemicals such a dichlorodiphenyl-trichloroethane (DOT), which was very effective globally before its ban because of adverse effects on bird populations. Malaria also is an outstanding example of ecogenetics, the interaction of environmental exposures with inherited susceptibility. Children and adults with hemoglobin $\mathrm{S}$ (sickle cell trait) have red blood cells less hospitable to the life stage of the malaria parasite that infects and propagates in the blood, compared with the red blood cells of individuals with normal $\mathrm{HbA}$. $\mathrm{HbS}$ individuals are more likely to survive the infection and go on to reproduce, transmitting their genes to the next generations. This is natural selection in action. Allison (1954) deduced that malaria was 
the selective factor maintaining the $\mathrm{HbS}$ gene in populations in the face of high mortality from sickle cell anemia when individuals received a double dose of the gene ( $\mathrm{HbSS}$ ). $\mathrm{HbC}, \mathrm{HbE}$, beta thalassemias, and glucose-6-phosphate dehydrogenase deficiencies fit this same pattern of "balanced polymorphisms" (Motulsky 1964). A 20\% increase in fitness for individuals with the trait could balance an $85 \%$ decrease in fitness of homozygous HbSS individuals (Gelehrter et al. 1998).

Phylogenetic analyses have radically revised our thinking about the origin of $P$. falciparum. For many years, the evidence seemed to point to co-speciation of $P$. falciparum in humans and Plasmodium reichenowi in chimpanzees, evolved separately from a presumed common ancestor over 5-7 Ma. That was based on a single isolate of $P$. reichenowi. Rich et al. (2009), with eight new isolates, showed that the global totality of $P$. falciparum strains is included within the much more diverse $P$. reichenowi variation. All extant $P$. falciparum populations seem to have originated from the parasite infecting chimpanzees by a single-host transfer about 10,000 years ago. Moreover, inactivation of the gene $\mathrm{CMAH}$ in the human lineage blocked conversion of the sialic acid $\mathrm{N}$-acetylneuraminic acid (5Ac) to N-glycolylneuraminic acid (Neu5Gc), making humans resistant to $P$. reichenowi.

Vivax malaria represents an entirely different mechanism for evolution of resistance and susceptibility. International studies of blood group antigens on red blood cells revealed that West African populations had a Duffy-negative phenotype and Fy-/Fy- genotype, which are rare among Caucasian and Asian populations. Fy-/Fy- individuals are completely resistant to $P$. vivax infection because the Fy blood group antigen is the receptor through which the $P$. vivax parasite enters erythrocytes. Whether this infection is sufficiently life-threatening to fully account for the ubiquitous presence of Fy- in these populations is not resolved (Vogel and Motulsky 1997; Omenn 2010).

HIV/AIDS The Duffy mechanism was a clue to explain the epidemiological observation that some men very highly exposed to the HIV/AIDS virus did not become infected. The most striking specific mechanism involves a mutant CCR5 receptor on lymphocytes (a 32 amino acid deletion). CCR5 is an essential component of the entry mechanism for $\mathrm{HIV}$; if there is no entry, there is no infection and there is no risk of transmission to others. We have no clue as to the natural selection driver for CCR5 mutations to accumulate in the human population (Heeney et al. 2006). We now know 20 polymorphisms of receptors, co-receptors, cytokine ligands, and HLA genes that influence susceptibility to HIV infection, replication, or relevant innate or adaptive immunity (Heeney et al. 2006). Viruses have a long history of co-evolution with molecules of the immune system. The presence of the CCR5 receptor seems to protect against West Nile virus; thus, we should be alert that development and public health use of inhibitors of CCR5 to reduce risk of HIV/AIDS could lead to increased risk of West Nile virusinduced encephalitis. Since the emergence of HIV/AIDS in the early $1980 \mathrm{~s}$, public interest in the origins of the HIV viruses has been intense; there is now strong evidence that HIV-1 and HIV-2 evolved from chimpanzees and from sooty mangabeys, respectively. Also, the human genome has a large array of endogenous retroviral sequences, suggesting a long history of co-evolution with retroviruses.

SARS and Influenza The coronavirus epidemic Severe Acute Respiratory Syndrome (SARS) appeared in southeast China and Hong Kong in 2002 and suddenly spread to Toronto via an air traveler. Modern molecular genetic epidemiologic methods swiftly identified the virus and permitted surveillance and control of this epidemic. The key link was humans handling infected animals. Global surveillance of highly exposed animal handlers is a strategy to identify such "emerging infections" at an early stage (Wolfe et al. 1998).

The best known viral infection linked through animal hosts is influenza. Influenza viruses are highly mutable and capable of rapid adaptation to selective factors in their environment, including vaccines. The H5N1 ("avian") and H1N1 ("swine") have quite different origins, with reassortment of strains in their animal hosts. One major barrier limiting cross-species transmission into humans (and vice versa) is the evolution of differences in sialic acid linkage binding specificity between humans and birds and humans and primates (Rich et al. 2009). Current research uses reconstituted influenza strains and reverse genetics to seek the specific genes and gene combinations that may drive virulence and host range; other researchers model the effects of vaccines and drugs on evolution and dynamics of the flu strains.

Microbiome Modern genomic analyses of bacteria DNA from organisms that are not readily cultured and grown in the laboratory have revealed remarkable co-existence of microbes in every ecosystem, including all the internal and external surfaces of our bodies. These complex microbial communities are called the "microbiome". There are an estimated ten times as many microbial cells as human cells in our bodies. They perform critical functions in digestion and host defenses. We provide unique habitats that have restricted colonization to a relatively small range of microorganisms. Our changing hygiene practices, diet, medical therapies, chemical exposures, and public health programs continue to cause changes in the microbiome (Turnbaugh et al. 2007).

Antibiotic Resistance/Evolution in Action Within the microbial world, there is interspecies competition and cooperation 
mediated through exchange of genetic material. Microbes compete for food and space, adapting to selective pressures. Fungi are particularly notable for evolution of antimicrobial chemical products that protect them against bacteria. Patients have benefitted from these antibiotics found in nature, starting with Fleming's use of an extract of Penicillium to kill Gram-positive bacteria. Microbes respond promptly to negative natural selection in the form of antibiotics by developing genetically transmitted resistance to the action of individual antibiotics or sets of antibiotics. Strains of Mycobacterium tuberculosis resistant to multiple drugs put healthcare workers at great risk; when these strains were recognized about 20 years ago, an aggressive public health campaign to identify and isolate such patients and ensure full dosage therapy with whatever agents still were effective contained the outbreaks. A similar challenge exists today with multiply resistant Staphylococcal aureus, acquired mostly in hospitals but increasingly in the community. Individuals immune-suppressed due to HIV infection, steroid treatments, cancer chemotherapy, or genetic immune deficiency disorders are particularly vulnerable to a broad range of infectious agents.

Vaccination has been a spectacularly effective public health intervention over the past 230 years. Modern vaccination approaches can be designed, in at least some situations, to select for less rather than more virulent strains or for other desired characteristics. For example, the diphtheria toxoid vaccine selects against toxin production, which is what causes disease, rather than against growth or survival of the Corynebacterium. Vaccination with the sevenconjugate vaccine against Streptococcus pneumonia has reduced carriage of penicillin-resistant serotypes but not invasive isolates (Karnezis et al. 2009). Bioinformatics tools, databases, and ontologies are helping researchers to organize information about immunization and to design new vaccination strategies (www.violinet.org/vaccineontology).

\section{Cultural Evolution-from Hunting and Gathering to Modern Agriculture and Dietary Practices}

Nutrition and diets are particularly salient subjects for modern public health courses, especially courses for nonmajors. Important aspects of anthropology, epidemiology, biochemistry, genetics, and toxicology need to be covered in an interdisciplinary fashion.

Origins, Evolution, and Consequences of the Western Diet Many of the diseases endemic in contemporary Western populations, and spreading across the globe, have arisen through discordance between our ancient geneticallyinfluenced biology and the dietary, cultural, and physical activity patterns of modern societies. Catchy titles like "Stone agers in the fast lane" (Eaton et al. 1988) set the stage. Food-processing procedures and different crops that were introduced during the Neolithic Period have altered fundamentally seven critical nutritional characteristics of ancient hominin diets: glycemic load, fatty acid composition, macronutrient composition, micronutrient density, acid-base balance, sodium/potassium ratio, and fiber content (Cordain et al. 2005). Modern diets are dominated by dairy products, refined cereal grains lacking germ and bran, refined sugars (sucrose and fructose), vegetable oils with low $\omega-3$ and high $\omega-6$ fatty acids, alcoholic beverages, salt, and $\omega-6$ saturated, fatty acid-rich mammalian meats. These foods displaced the wild plant and animal foods of our predecessors. Cordain et al. call our diet "the evolutionary collision of our ancient genome with the nutritional qualities of recently introduced foods". This collision is manifested by many chronic diseases that dominate public health concerns: obesity, diabetes, cardiovascular disorders, high blood pressure, dyslipidemias, osteoporosis, several cancers, and bowel, inflammatory, and autoimmune diseases. All remain rare among contemporary hunter-gatherer societies. Modern foods are also net acid generators, compared with net baseproducing pre-agricultural diets; the latter tended to protect against osteoporosis, muscle wasting, calcium kidney stones, high blood pressure, and exercise-induced asthma (Frassetto et al. 2001).

The Emergence of Dairy-Based Societies and Lactose Tolerance Besides the changes above, about 7,000-10,000 years ago progressive domestication of sheep, goats, and then cattle occurred in many places; about 6,000 years ago ingestion of the milk from these animals became a part of the human diet (Vogel and Motulsky 1997). Milk contains casein protein, calcium salts, water, and lactose (galactose-glucose disaccharide sugar). The ability to digest lactose declines rapidly in most humans after weaning because of a normal decline in the activity of the intestinal enzyme lactase. Before the drinking of non-human milk, there was no further need for this enzyme after weaning ended the dependence on mother's milk. Populations with a long history of cattle domestication and milk drinking selected for the "persistence of lactase" trait. It is an interesting question which phenotype is "normal", intolerance or tolerance for lactose sugar after weaning. The prevalence of persistence of lactase (lactose tolerance) has reached $>90 \%$ among northern Europeans (Swedes), $50 \%$ in Spanish and Arab populations, 5-20\% among African populations, and only $1 \%$ among Chinese and Native Americans. This genetic variation is a source of health disparities in public health nutrition programs.

There are many interesting evolutionary questions about lactase persistence. How many times has a mutation 
occurred that was then selected positively to reach high prevalence today-once, twice, many times? How did the mutation or mutations spread globally? What is the mechanism for what seems to be a regulatory on/off mutation? Why are symptoms of lactose intolerance so variable within human populations? See Tishkoff et al. (2007), Hollox and Swallow (2002), and Itan et al. (2009).

We must always consider that a trait of interest may be adaptive for more than one reason and may therefore be selected for some other or additional benefit to reproduction and survival. In addition to protein, calcium, and sugar, milk provides water, which is especially important in arid regions, whereas lactose intolerance leads to water loss via diarrhea.

The social and public policy context of "nutrigenomics" can be illustrated with lactose intolerance. The dairy industry has had a long-running successful campaign with "Got Milk?" advertisements. Originally, the tag line was "Everybody needs milk." The Najavo Indian Nation painted their adobes with federal surplus powdered milk; the unkind comments of outsiders reflected ignorance of the unpleasant gastrointestinal symptoms the milk caused in these people, of whom $>95 \%$ were lactose intolerant.

In response to objections on behalf of nonwhite U.S. populations, the tagline was changed to "Milk has something for everybody." This case stimulates us to realize that "the normal state" depends on time and place and environmental conditions. Factors other than the primary gene variant contribute to variation in severity of symptoms, making population testing to identify susceptible individuals before they are symptomatic much more complex than one might think. Many cases of irritable bowel syndrome might be attributable to this condition. (For other aspects of evolution, diet, and public health; see Omenn 2010).

\section{Excessive Hemostasis and Thrombosis}

Ischemic atherosclerotic heart disease, leading to myocardial infarction (heart attack), strokes, or heart failure, is a relatively recent phenomenon for humans. The pathology of myocardial infarction was described first by Herrick in 1912 (Herrick 1983). The first textbook on heart attacks was published by Paul Dudley White (1931). A key element of normal cardiovascular function is hemostasis. For animals in the wild and for hunter-gatherers, the risk of bleeding to death after an injury is a strong evolutionary pressure. Likewise, women experienced pressure to have greater hemostasis to survive pregnancy-related hemorrhages. Those individuals with more active hemostasis were more likely to survive to sexual maturity and pass on their genes (Coller 1997; Ding and Kullo 2009). At the same time, there was little selective pressure against excessive hemostasis and thrombotic disorders, since heart attacks and strokes occur typically after the active reproductive years.

Understanding these physiological and evolutionary dynamics, it was feasible to develop a strategy for a new pharmaceutical agent and its molecular target. Successful therapies have been directed at platelet glycoproteins (Coller 1997). These molecules are biosensors and mediators of integrin receptor modulation, cell/cell adhesion, and thrombus formation. Platelets are deposited on damaged atherosclerotic plaques together with deposition of fibrin and thrombin and binding of a large protein called von Willebrand factor (vWf), collagen, and tissue factor, culminating in occlusive thrombus formation. The platelet GPIIb/IIIa receptors on the luminal surface of the adherent platelets are activated and undergo a conformation change that enhances their binding of plasma fibrinogen, $\mathrm{vWf}$, and other glycoproteins.

The first anti-integrin receptor drug, an antagonist of the platelet glycoprotein GPIIb/IIIa, was a mouse/human chimeric monoclonal antibody (c7E3 Fab; abciximab; ReoPro) approved by the Food and Drug Administration in the U.S. in 1994. It was safe and effective in reducing the risk of ischemic complications of atherosclerotic coronary and cerebrovascular disease (heart attacks, sudden death, and strokes). Coller (1997) emphasized that this drug is a leading example of rational drug design - identifying adhesion molecules as therapeutic targets and then producing successive generations of effective inhibitors that meet the stringent safety standards for drug approval. The evolutionary pressure for life-saving hemostasis led to notable redundancy in our hemostatic systems; thus, treatments of patients with anticoagulants, antiplatelet agents, or thrombolytic agents have excellent safety profiles. Moreover, this glycoprotein is expressed only in megakaryotypes (platelet precursors) and platelets, so it is truly a specific target. Reflecting the need for instantaneous response to hemorrhage, the density of GPIIb/IIIa receptors on platelet surfaces is about 80,000 copies per platelet less than $200 \mathrm{~A}$ apart, with an additional internal pool of receptors in alpha granules. These receptors are activated by molecular signals of vessel damage, followed later by a complex process of inactivation. A vast pharmacologic and clinical literature supports our understanding of this highly evolved, complex system of cell adhesion, responses to hemorrhage, and thrombotic and antithrombotic effects.

Ding and Kullo (2009) provide a broad review of apparent positive, balancing, and purifying (negative) selection in relation to the many risk factors for coronary heart disease. Besides hypercoagulability, blood pressure regulation, inflammation, lipoprotein and glucose metabolism, metabolic syndrome, and oxidative stress are examined in light of climatic and dietary changes and host response to 
pathogens. The recognition of limited variation within haplotypes to identify recent positive selective pressure is illustrated with SNP 44 of Calpain-10, involved in regulation of blood glucose levels (Vander Molen et al. 2005). The sodium hypothesis, that sodium-conserving mechanisms conferred a survival advantage among human populations in the hot, humid climate of Africa, but then predisposed to high blood pressure in temperate climates, is wellsupported. The newly-discovered regulator of the lowdensity lipoprotein receptor, PCSK9, has multiple interesting evolutionary features; it is also a prime target for new drug development. Many other associations with coronary heart disease (e.g., factor V Leiden) lack explanations tied to natural selection or are quite speculative.

\section{Global Climate Change}

The global environment is a popular topic for undergraduates and graduate students from many majors and disciplines. Global average surface temperatures have increased $0.8^{\circ} \mathrm{C}\left(1.4^{\circ} \mathrm{F}\right)$ over the past century, mostly in the past 30 years, with a driver to further increases from carbon dioxide already accumulated in the atmosphere. We can expect an increasing focus on modeling and predicting coevolution of humans and many relevant plant, microbial, invertebrate, and vertebrate species under the selective forces of global climate change and our attempts to mitigate and adapt to climate change. Geographic shifts with temperature and humidity will have major effects on agriculture, vector-borne diseases, and migrations of populations (Last 1993; Ebi and Semenza 2008). The Arctic is particularly susceptible to climate change, with warming occurring at a rate twice that of moderate zones, leading to striking changes in the forests and viability of crops, appearance of unfamiliar insects and microbes, and thinning and breakup of the arctic ice. We can anticipate progressive major changes in temperature, humidity, habitats, vectors, and transmission for a host of infectious agents. Seasonality is another important climate variable for infectious diseases, recognized since the time of Hippocrates. Seasonality produces alternating periods of high transmission and population bottlenecks that limit strain diversity and cause rapid genetic shifts. Models show that small seasonal changes in host-pathogen dynamics, including host social behavior and contact rates, may be sufficient to create large seasonal surges in disease incidence, which are likely to become more extreme with climate change.

The term adaptation in the climate change literature is analogous to the term prevention in public health, ranging from reduction of greenhouse gas emissions to redesign of cities to minimize heat islands and heat waves, to surveillance for diseases like tickborne Lyme disease and animal- to-human transmission of exotic new infections, or to mitigation of health disparities in human impacts of rising sea and river levels. Public health professionals will be critical to adaptation strategies, hopefully informed by an evolutionary perspective about the interrelations between living things and their environments.

\section{Closing Comment}

Important examples of practical applications of evolutionary understanding in modern public health include obesity, influenza, and appropriate uses of antibiotics. As documented by First Lady Michelle Obama, the Surgeon General, and the Centers for Disease Control, the prevalence of obesity and overweight has increased sharply in the past 30 years, with huge consequences for the burden of chronic diseases and health care costs. The global epidemic of obesity represents a combination of rapidly changing, culture-based behavior changes and discordant genomic predispositions that cannot be ignored. Meanwhile, we face simultaneous influenza epidemics from seasonal H1N1 strains and from lingering or reappearing $\mathrm{H} 5 \mathrm{~N} 1$ avian flu strains. Finally, we remain in an arms race with bacteria whose environments inside us and around us we are constantly changing, stimulating their own rapid evolution.

Evolution, natural selection, and population dynamics act over very long periods of time. We have learned in recent years, as highlighted by Science magazine's "Breakthrough of the Year 2005," that we can actually observe "evolution in action"-in the Galapagos, the Arctic and Antarctic, hospitals, rural and urban waste streams, and many other settings, with impacts on public health and implications for our public health research agenda. We can be confident that evolutionary perspectives will provide a useful foundation for research and communication in public health (Nesse and Stearns 2008; Omenn 2010) as well as in medical care (Williams and Nesse 1991; AAMC-HHMI 2009; Nesse et al. 2010; Nesse 2011).

Acknowledgements This article is based on a presentation at The Evolution Education Symposium in Honor of George Willliams at Norman, OK, USA on 19 June 2011.

\section{References}

AAMC-HHMI Scientific Foundation for Future Physicians Committee. Scientific foundations for future physicians. Washington: American Association of Medical Colleges and Howard Hughes Medical Institute; 2009.

Allison AC. Protection afforded by sickle cell trait against subtertian malarial infection. Br Med J. 1954;1:290-4. 
Coller B. Perspectives series: cell adhesion in vascular biology. Platelet GPIIb/IIIa antagonists: the first anti-integrin receptor therapeutics. J Clin Investig. 1997;99:1467-71.

Cordain L, Eaton SB, Sebastian A, Mann N, Lindeberg S, Watkins BA, O'Keefe JH, Brand-Miller J. Origins and evolution of the Western diet: health implications for the 21st century. Am J Clin Nutr. 2005;81:341-54.

Ding K, Kullo IJ. Evolutionary genetics of coronary heart disease. Circulation. 2009;119:459-67.

Eaton SB, Konner M, Shostak M. Stone agers in the fast lane: chronic degenerative diseases in evolutionary perspective. Am J Med. 1988;84:739-49.

Ebi KL, Semenza JC. Community-based adaptation to the health impacts of climate change. Am J Prev Med. 2008;35:501-7.

Ewald PW. Evolution of virulence. Infect Dis Clin North Am. 2004;18:115.

Frassetto L, Morris Jr RC, Sellmeyer DE, Todd K, Sebastian A. Diet, evolution and aging - the pathophysiologic effects of the postagricultural inversion of the potassium-to-sodium and base-tochloride ratios in the human diet. Eur J Nutr. 2001;40:200-13.

Gelehrter T, Collins F, Ginsburg D. Principles of medical genetics. 2nd ed. Baltimore: Williams \& Wilkins; 1998. p. 51.

Gluckman P, Beedle A, Hanson M. Principles of evolutionary medicine. Oxford: Oxford University Press; 2009. p. 296.

Heeney JL, Dalgleish AG, Weiss RA. Origins of HIV and the evolution of resistance to AIDS. Science. 2006;313:462-6.

Herrick JB. Landmark article (JAMA 1912), Clinical features of sudden obstruction of the coronary arteries. JAMA. 1983;250:1757-65.

Hollox EJ, Swallow DM. In: King RA, Rotter JI, Motulsky AG, editors. The genetic basis of common diseases. Oxford: Oxford University Press; 2002. p. 250-65.

Itan Y, Powell A, Beaumont MA, Burger J, Thomas MG. The origins of lactase persistence in Europe. PLOS Comput Biol. 2009;5:e1000491.

Karnezis TT, Smith A, Whittier S, Haddad Jr J, Saiman L. Antimicrobial resistance among isolates causing invasive Pneumococcal disease before and after licensure of heptavalent conjugate Pneumococcal vaccine. PLoS One. 2009;4:e5965.

Laporte RE, Omenn GS, Serageldin I, Cerf VG, Linkov F. A scientific supercourse. Science. 2006;312:526.

Last JM. Global change: ozone depletion, greenhouse warming, and public health. Annu Rev Public Health. 1993;14:115-36.
Motulsky AG. Hereditary red cell traits and malaria. Am J Trop Med Hyg. 1964;13(Suppl):147-58.

Nesse RM. Ten questions for evolutionary studies of disease vulnerability. Evol Appl. 2011;4(2):264-77.

Nesse RM, Stearns SC. The great opportunity: evolutionary applications to medicine and public health. Evol Appl. 2008;1:28-48.

Nesse RM, Bergstrom CT, Ellison PT, Flier JS, Gluckman P, Govindaraju DR, Niethammer D, Omenn GS, Perlman RL, Schwartz MD, Thomas MG, Stearns SC, Valle D. Making evolutionary biology a basic science for medicine. Proc Natl Acad Sci U S A. 2010;107 Suppl 1:1800-7.

Omenn GS. Evolution and public health. Proc Natl Acad Sci U S A. 2010;107 Suppl 1:1702-9.

Rich SM, Leendertz FH, Xu G, Lebreton M, Djoko CF, Aminake MN, Takang EE, Diffo JL, Pike BL, Rosenthal BM, et al. The origin of malignant malaria. Proc Natl Acad Sci U S A. 2009;106:14902-7.

Stearns SC, Koella JC, editors. Evolution in health and disease. 2nd ed. New York: Oxford University Press; 2007.

Tishkoff SA, Reed FA, Ranciaro A, Voight BF, Babbitt CC, Silverman JS, Powell K, Mortensen HM, Hirbo JB, Osman M, Ibrahim M, Omar SA, Lema G, Nyambo TB, Ghori J, Bumpstead S, Pritchard JK, Wray GA, Deloukas P. Convergent adaptation of human lactase persistence in Africa and Europe. Nat Genet. 2007;39:31-40.

Trevathan W, Smith EO, McKenna JJ, editors. Evolutionary medicine and health: new perspectives. New York: Oxford University Press; 2008.

Turnbaugh PJ, Ley RE, Hamady M, Fraser-Liggett CM, Knight R, Gordon JI. The human microbiome project. Nature. 2007;449:804-10.

Vander Molen J, Frisse LM, Fullerton SM, Qian Y, Del Bosque-Plata L, Hudson RR, Di Rienzo A. Population genetics of CAPN10 and GPR35: implications for the evolution of type 2 diabetes variants. Am J Hum Genet. 2005;76:548-60.

Vogel F, Motulsky AG. Human genetics: problems and approaches. 3rd ed. Berlin: Springer; 1997.

White PD. Heart disease. Birmingham, Alabama: Gryphon Press, Classics of Medicine Library; 1931.

Williams GC, Nesse RM. The dawn of Darwinian medicine. Q Rev Biol. 1991;66:1-22.

Wolfe ND, Escalante AA, Karesh WB, Kilbourn A, Spielman A, Lal AA. Wild primate populations in emerging infectious disease research: the missing link? Emerg Infect Dis. 1998;4:149-58. 